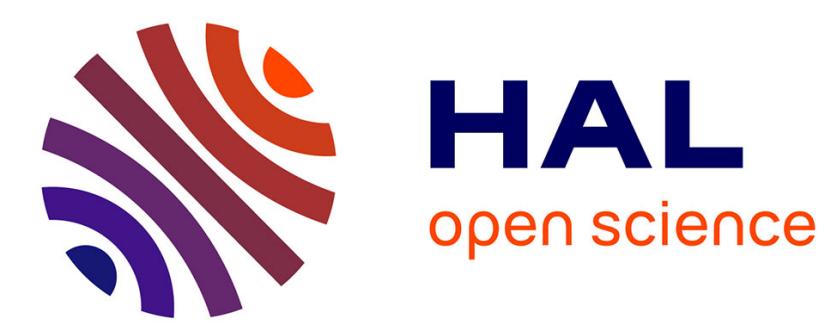

\title{
Dynamic characteristics of circular CMUTs with air-filled cavities
}

Ayrat Galisultanov, Patrice Le Moal, Vincent Walter, Gilles Bourbon

\section{To cite this version:}

Ayrat Galisultanov, Patrice Le Moal, Vincent Walter, Gilles Bourbon. Dynamic characteristics of circular CMUTs with air-filled cavities. International Ultrasonics Symposium, Sep 2016, Tours, France. hal-03107834

\section{HAL Id: hal-03107834 \\ https://hal.science/hal-03107834}

Submitted on 12 Jan 2021

HAL is a multi-disciplinary open access archive for the deposit and dissemination of scientific research documents, whether they are published or not. The documents may come from teaching and research institutions in France or abroad, or from public or private research centers.
L'archive ouverte pluridisciplinaire HAL, est destinée au dépôt et à la diffusion de documents scientifiques de niveau recherche, publiés ou non, émanant des établissements d'enseignement et de recherche français ou étrangers, des laboratoires publics ou privés. 


\title{
Dynamic Characteristics of Circular CMUTs with Air-Filled Cavities
}

\author{
A. Galisultanov, P. Le Moal, V. Walter, G. Bourbon \\ Institut FEMTO-ST, UMR CNRS 6174, \\ University Bourgogne Franche-Comté (UBFC) \\ 25000, Besançon, France \\ ayrat.galisultanov@femto-st.fr
}

\begin{abstract}
Our work deals with the study of the dynamic characteristics of a single circular CMUT transducer under squeeze film damping effect. A simplified analytical approach is developed for parallel plate approximation for the electromechanical behavior and the squeeze film damping. Analytically calculated results demonstrates, that trends of resonant frequency with bias voltage are defined by the ratio of air spring constant to mechanical spring constant. At the same time FEM multiphysics model is built to define the influence of membrane flexibility and venting boundary conditions to the dimensionless viscous and elastic damping forces. FEM study shows the presence of viscous loss in the sealed air-filled cavity and the insensitivity of squeeze film damping forces to the type of lateral venting boundary conditions in high squeeze number region which is typical for CMUT operation. Finally, the experimental dynamic characterization is conducted on CMUT with $67 \mu \mathrm{m}-167 \mu \mathrm{m}$ radii fabricated utilizing an anodic bonding technology. Experimental data are in a good agreement with the results of multiphysics FEM calculation, which take into account bias voltage, initial plate deflection, radiation loss and squeeze film damping.
\end{abstract}

Keywords—vented CMUT; squeeze film damping; bias voltage; resonant frequency; bandwidth

\section{INTRODUCTION}

Typical capacitive micromachined ultrasound transducers (CMUTs) consist of metallized movable membrane separated from the substrate with bottom electrode by the gap. In most cases bottom electrode is covered by insulation layer to prevent breakdown in collapse point. The top of the movable membrane can be loaded by fluid or gas and the membrane vibration is used for ultrasound waves transmission and receiving for immersion [1] and air coupled [2-3] applications, respectively.

The presence of gas in narrow CMUT cavity leads to squeeze film damping [4] and affects the dynamic performances of vented and sealed CMUT. The viscous damping force for parallel plate motion (piston model) presents only for open venting lateral boundary conditions, and caused by gas flow in and out of the cavity [5]. For the case of sealed CMUT cavity and parallel vibrated rectangular plates Darling et al. [6] demonstrated pure spring force without viscous damping. However, piston model does not take into account membrane flexibility and proper lateral venting boundary conditions at the perimeter of the CMUT cavity most part of which is sealed [2,7]. Analytical approach for modeling of flexible microstructures under the effect of squeeze film damping has been developed in [8]. In [9] the authors supposed that elastic deflection of the upper plate for sealed structure leads to viscous damping. Ahmad et al. [10] have obtained an analytical solution of the Reynolds equation for air flow inside a completely sealed circular air cavity, taking into account the elastic deformation of the vibrating membrane. They have showed that the resulting flow produces an air spring force without dissipation.

Most of CMUTs applications require high bias voltage in order to improve either the sensitivity of the receiver mode or the radiated acoustic pressure of the transmitter mode. Such high bias voltage strongly affects the dynamic characteristics of CMUTs in terms of the resonant frequency. In particular, the "stiffening" effect of the squeeze air film in the back cavity and the electrostatic "softening" effect lead to contradictory influences on the resonant frequency.

In the presented work a simplified analytical approach is developed with parallel plate approximation for the electromechanical behavior and the squeeze film effects. At the same time, a FEM multiphysic model based on the NavierStokes equations is built to define the influence of membrane flexibility and venting boundary conditions to dimensionless viscous and elastic damping forces. Finally, the experimental dynamic characterization is conducted on CMUT with 67 $167 \mu \mathrm{m}$ radii fabricated utilizing an anodic bonding technology.

\section{Circular CMUT with AIR-FILled CAVITY}

Consider electrostatically actuated circular piston of radius $a$ and thickness $t$ which is suspended above uniform air-filled cavity with thickness $g_{0}$ of the same radius. The spring constant of piston is $k_{s}$. The top of the piston is metalized, the electrode thickness is neglected. The bottom electrode is protected by insolation layer of thickness $i$. The relative dielectric coefficients of the insulation layer and the plate are $\mathcal{E}_{i}$ and $\varepsilon_{t}$, respectively. Mass of piston equals to $m=\rho t S$, where $\rho$ is piston's material density, $S=\pi a^{2}$ is the area of circular piston.

Parallel plate transducer model for evacuated CMUT has been developed successfully in [11]. When the bias voltage $V_{D C}$ is applied between the two electrodes, the electrostatic attractive force causes piston static displacement $x$. The 
displacement can be found as a solution of equilibrium equation of electrostatic and mechanical restoring forces:

$$
\frac{\varepsilon_{0} S V_{D C}^{2}}{2\left(d_{e f f}-x\right)^{2}}=k_{S} x
$$

where $\varepsilon_{0}$ is the permittivity of vacuum, $d_{e f f}=g_{0}+t / \varepsilon_{t}+i / \varepsilon_{i}$ is the effective electrostatic gap distance. The displacement for collapse voltage $V_{c o l}$ is one-third of the effective gap.

Superposition of DC bias voltage $V_{D C}$ and small signal $V_{A C}$ bias voltage applied to CMUT electrodes leads to membrane vibration in a frequency of $\mathrm{AC}$ excitation. The behavior of a trapped gas film between two parallel vibrated plates can be found as solution of linearized Reynolds equation for compressible gas [4]. Linearized Reynolds equation neglects fluid inertia and uses the isothermal condition. A solution for circular plates with open boundaries in parallel motion has been given by Blech [12]. He has found the expressions for dimensionless viscous $f_{d}(\sigma)$ and elastic $f_{e}(\sigma)$ damping force as a function of squeeze number $\sigma$ :

$$
\sigma=\frac{12 \mu \omega a^{2}}{P_{a} g_{0}^{2}}
$$

where $\mu$ is the viscosity of the air, $\omega$ is the radial frequency of vibration, $P_{a}$ is the ambient pressure. In case of low frequency oscillation and consequently low squeeze number, plate moves with low velocity and the gas has time to "leak" out from the gap. In this case viscous damping force dominates. In the opposite case of high frequency oscillation, the gas film is mainly compressed and has no time to "leak". In this case the elastic damping force dominates and the air film acts as a spring.

The dynamic behavior of the parallel plate circular transducer with air-filled cavity is defined by the differential equation of motion:

$$
m \ddot{x}+c_{d} \dot{x}+\left(k_{s}+k_{e}\right) x=F_{0} \sin (\omega t)
$$

where $F_{0}$ is the amplitude of small signal harmonic excitation, $c_{d}=P_{a} S /\left(\omega g_{0}\right) \cdot f_{d}(\sigma)$ is the coefficient of viscous damping force, $k_{e}=P_{a} S / g_{0} \cdot f_{e}(\sigma)$ is the air spring constant. In this equation we assumed the absence of load on the top of movable piston. For standard CMUTs dimensions and operating frequencies the squeeze number is large and we can

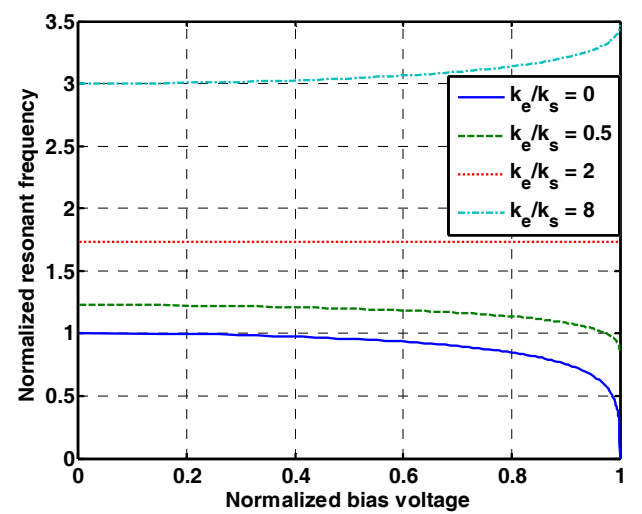

Fig. 1. The dependence of normalized frequency on normalized bias voltage for different $k_{e} / k_{s}$ ratio use pure spring assumption for CMUT with open cavity: $f_{d}(\sigma) \approx 0$ and $f_{e}(\sigma)=1$ [4]. For sealed cavity and parralel plate motion Darling et al. [6] have demonstrated pure spring force with no viscous damping for parallel vibrated rectangular plates. We assumed the same behavior for sealed circular plate., i.e. $f_{d}(\sigma)=0$ and $f_{e}(\sigma)=1$.

For high values of $\sigma$, air spring constant is a function of gap size only, and, consequently, of the bias voltage level. Therefore DC bias voltage causes both the electrostatic "softening" effect and the "stiffening" effect of the squeeze air film in the back cavity. In assumption of relatively thin insulation and membrane layer $\left(d_{e f f} \approx g_{0}\right)$ the total stiffness $k$ for CMUT with vented cavity is:

$$
k=k_{s}+k_{e}+\left(k_{e}-2 k_{s}\right) \cdot x /\left(g_{0}-x\right)
$$

Equation (4) can be also used for the case of sealed CMUT for stable cavity pressure assumption.

The dependence of normalized frequency $\omega_{n} / \omega_{0}$ on normalized DC bias voltage $V_{D C} / V_{c o l}$ for different $k_{e} / k_{s}$ ratio presented in Fig. 1. Here $\omega_{n}$ and $\omega_{0}$ are natural frequency of CMUT for the case of vented and evacuated cavity, respectively. The curves are calculated from Eq. (1) and (4). The analysis of results shows that for $k_{e} / k_{s}=2$ the natural eigenfrequency of piston vibration does not depend on applied DC bias voltage and equals to $\sqrt{3} \omega_{0}$. The resonant frequency of CMUT cell with air-filled cavity in the vicinity of collapse point is mainly defined by the air spring constant and tends to $\left(3 k_{e} / 2 m\right)^{1 / 2}$.

\section{FEM ANALYSIS OF CMUT WITH FLEXIBLE PLATE}

The single degree-of-freedom model does not take into account plate flexibility, gap non-uniformity and proper lateral venting boundary conditions. In this part of our work we develop FEM model of flexible plate under the effect of squeeze-film to obtain the dimensionless viscous and elastic damping forces.

The simplified axisymmetric model is developed in COMSOL Multiphysics package. Navier-Stokes equation within COMSOL Thermoacoustics interface is used to model air-filled cavity. In our analysis we use two extreme types of lateral venting boundary conditions: open and sealed. For both cases we suppose that the pressure in the cavity $P_{a}$ is stable and equals to one atmosphere $(101.3 \mathrm{kPa})$. The radius and the thickness of clamped circular silicon plate are $150 \mu \mathrm{m}$ and 2.3 $\mu \mathrm{m}$, respectively. The air-filled gap thickness $\left(g_{0}\right)$ is varied from 1 to $40 \mu \mathrm{m}$ and rarefaction effect is not taken into account. The displacement response of the system is calculated in Frequency Domain Study. The excitation signal is uniform pressure applied to the top of plate.

The difference between the natural resonant frequency of plate vibrating over a uniform thin air gap $\omega_{n}$ and the resonant frequency of the same plate in vacuum $\omega_{0}$ is defined by air spring constant:

$$
k_{e}=m\left(\omega_{n}{ }^{2}-\omega_{0}{ }^{2}\right)
$$

From (5) the dimensionless elastic damping force can be find as (6). As soon as the top of the movable membrane is not 


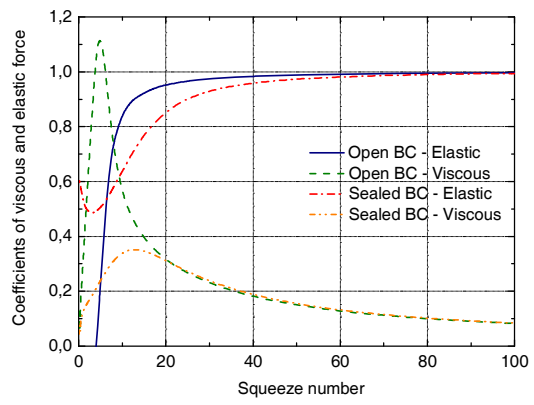

Fig. 2. FEM calculated dependence of dimensionless viscous and elastic damping force on squeeze number for open and sealed cavity
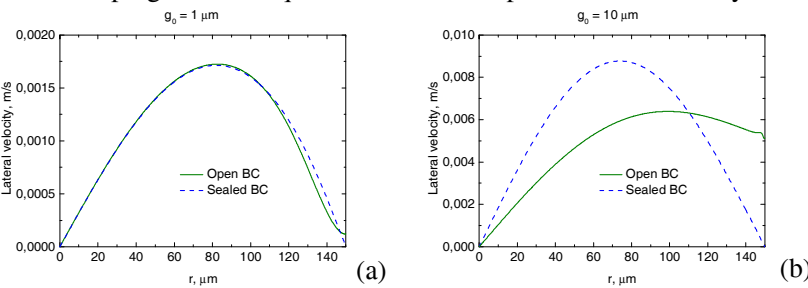

Fig. 3. The lateral velocity profile along the center of open and sealed cavity in the vicinity of resonant frequency

loaded by fluid or gas, the bandwidth $\Delta \omega$ of this system is determined by squeeze film damping. The dimensionless viscous damping force can be defined from (7).

$$
\begin{aligned}
& f_{e}(\sigma)=\frac{k_{e}}{P_{a} S / g_{0}}=\frac{m\left(\omega_{n}^{2}-\omega_{0}^{2}\right)}{P_{a} S / g_{0}} \\
& f_{d}(\sigma)=\frac{\Delta \omega}{\omega_{n}} \cdot \frac{m \omega_{n}^{2}}{P_{a} S / g_{0}}
\end{aligned}
$$

The FEM calculated (6)-(7) dependencies of the dimensionless viscous and elastic damping force on squeeze number for open and sealed cavity are presented in Fig. 2. Squeeze film number is defined from (2). The analysis of FEM obtained data shows that the dimensionless elastic damping force coefficient tends to 1 for high squeeze number for both types of venting boundary conditions. Consequently, the air spring constant equals to $P_{a} S / g_{0}$. In the case of small squeeze number the frequency difference is mainly defined by air mass load. This means that obtained value do not accurately predict dimensionless elastic damping force coefficient value, which has to tend to zero for infinitesimal squeeze number.

Fig. (2) demonstrates the presence of viscous loss in sealed air-filled cavity. The damping pressure amplitude is nonuniform due to non-uniform amplitude of velocity along of vibrating plate. This pressure difference causes air flow inside the sealed cavity (Fig. 3), and, consequently, viscous loss. The dimensionless viscous damping force for sealed and open cavity is the same in the case of high squeeze number, typical for CMUT operation. This results from the same gas velocity profile inside the cavity for both type of venting boundary conditions [Fig. 3 (a)]. In high squeeze number region the dimensionless viscous damping force coefficient is proportional to $\sigma^{-0.89}$. The gap increasing leads to increasing of the air flow in and out of open cavity (Fig. 3). Consequently, viscous loss component becomes higher for open cavity than for sealed cavity. The viscous loss mechanism of flexible plate and piston under squeeze film damping is completely different and defined by air motion profile inside the cavity.

The non-sensitivity to type of venting boundary condition allows us to use axisymmetric model for calculation of the real CMUT design with non-axisymmetric venting cavity. In further bandwidth and frequency calculation we do not take into account geometrical parameters of lateral venting channels.

\section{EXPERIMENTAL RESULTS AND DISCUSSION}

Fabrication of CMUTs has been performed using anodic bonding of a fixed thickness monocrystalline silicon layer (100) of a SOI wafer on a borosilicate glass substrate. The detailed process flow can be found in [7]. CMUT cells have been fabricated with four different radii $67 \mu \mathrm{m}, 87 \mu \mathrm{m}, 117$ $\mu \mathrm{m}$, and $167 \mu \mathrm{m}$. The silicon plate and the top aluminum electrode have the thickness of $2.3 \mu \mathrm{m}$ and 0.22 $\mu \mathrm{m}$, respectively. The initial air-filled gap between plate and bottom electrode is $0.75 \mu \mathrm{m}$. The bottom electrode thickness is $0.25 \mu \mathrm{m}$. The CMUT cavity is vented through lateral channel to atmosphere. It has been found that the center of the plate has non-zero deflection resulting from relaxation of the residual stress. This stress appears during fabrication process steps: wafers bonding, $\mathrm{Si}$ film release and aluminum deposition on top of the plate. The typical measured characteristics of fabricated CMUTs are presented in Table 1.

The displacement response as a function of the bias voltage has been measured using a Polytec laser vibrometer with vibrometer sensor head OFV-534 and an OFV-5000 controller (Fig. 4). The resonance frequency decreases with increasing bias voltage for CMUT with the radii $67 \mu \mathrm{m}$ and 87 $\mu \mathrm{m}$ (mechanical spring constant dominates). The analytical prediction of stable resonance frequency with the bias voltage is experimentally confirmed for CMUT with the radius of 117 $\mu \mathrm{m}$. For CMUT with the radius of $167 \mu \mathrm{m}$ resonance frequency increases with increasing bias voltage (air spring constant dominates).

The bandwidth increases with bias voltage for CMUT with the radii of $67 \mu \mathrm{m}$ and $87 \mu \mathrm{m}$, decreases for CMUT with the radius of $167 \mu \mathrm{m}$ and remains stable CMUT with the radius of $117 \mu \mathrm{m}$. For CMUT with the smallest radius experimentally
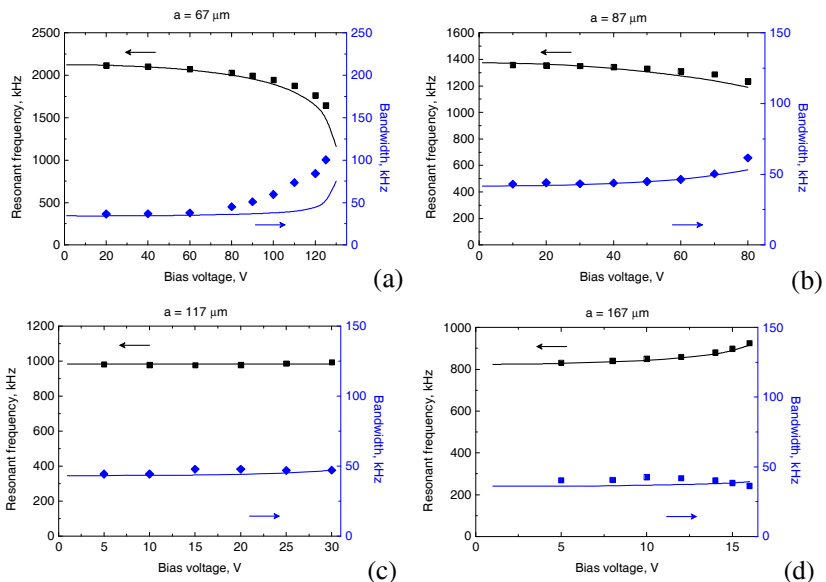

Fig. 4. Experimentally measured (*) and FEM calculated (-) the resonant frequency and the bandwidth on the bias voltage 
observed bandwidth increases from $36.5 \mathrm{kHz}\left(V_{D C}=1 \mathrm{~V}\right)$ to $100.2 \mathrm{kHz} \quad\left(V_{D C}=125 \mathrm{~V} \approx 0.95 V_{c o l}\right)$. Air-filled cavity both increases the bandwidth and provides a way for effective bandwidth control using bias voltage variation.

TABLE I. EXPERIMENTAL RESULTS

\begin{tabular}{|c|c|c|c|}
\hline $\begin{array}{c}\text { Plate radius, } \\
\boldsymbol{\mu m}\end{array}$ & $\begin{array}{c}\text { Bottom electrode } \\
\text { radius, } \boldsymbol{\mu m}\end{array}$ & $\begin{array}{c}\text { Static } \\
\text { deflection, } \mathbf{n m}\end{array}$ & $\begin{array}{c}\text { Collapse } \\
\text { voltage, } \mathbf{V}\end{array}$ \\
\hline 67 & 40 & 80 & 132 \\
\hline 87 & 56 & 160 & 83 \\
\hline 117 & 80 & 277 & 34 \\
\hline 167 & 120 & 80 & 16 \\
\hline
\end{tabular}

The bandwidth of the vented CMUT is defined by the squeeze film damping in back cavity and the radiation loss. The simplified axisymmetric model is developed in COMSOL Multiphysics software for dynamic response calculation of a single CMUT cell. In the model we use sealed venting boundary condition and neglect the lateral venting channel. For the radiation loss definition we load the top of the plate by the air. The perfectly matched layer is added to emulate an open and non-reflecting infinite domain. The measured geometrical parameters of the fabricated devices are accepted as a model parameters. Viscosity constant is replaced by effective viscosity constant [13] for initial gap size $0.75 \mu \mathrm{m}$. Silicon membrane has relatively high conductivity (1-30 $\Omega \cdot \mathrm{cm}$ ), so the effective top electrode is located at the bottom side of the plate. We combine COMSOL Thermoacoustics and Electromechanics interfaces and use two step Stationary Frequency Domain, Perturbation Studies. In Stationary Study we take into account residual stress, which causes static deflection of the plate and the bias voltage. At the second step (Frequency Domain, Perturbation Study) we apply small electrostatic excitation signal and define the resonant frequency and the bandwidth.

FEM calculated evolution of the resonance frequency and the bandwidth as a function of the bias voltage are presented in Fig. 4. The FEM model precisely defines the resonant frequency and the collapse voltage. Bandwidth for the low bias voltage is also precisely determined. This matching verifies the proposed approach of replacing of the real cavity of CMUT with non-axisymmetric lateral venting channels by axisymmetric sealed cavity. The FEM model does not correctly define the bandwidth for high bias voltage level, especially for CMUT with the radius of $67 \mu \mathrm{m}$. We suppose that relatively high value of error is determined by inaccurate effective viscosity value. Used in the model Verola et al. [14] approximation has used data from the experiments with the parallel plate motion. In our case the gap is non-uniform and very small. To describe such fluid film rarefied fluid flow equation is need [14].

\section{CONCLUSIONS}

Analytical calculations based on piston transducer model demonstrate, that dependence of the resonant frequency on the bias voltage is defined by the ratio of air spring constant to mechanical spring constant. The FEM analysis has shown the presence of the viscous damping for sealed cavity. Additionally, in the high squeeze number region, which is typical for CMUT operation, the dimensionless viscous and elastic damping force are insensible to the type of the lateral venting boundary conditions.

Finally, the experimental dynamic characterization is conducted on fabricated CMUT. The analytical prediction of resonant frequency behavior on bias voltage has been confirmed. Experimental data have been in a good agreement with the result of the multiphysics FEM calculation, which has taken into account the bias voltage, the initial plate deflection, the rarefaction effect, the squeeze film damping, and the radiation loss. This matching has been verified the proposed approach of replacing of the real cavity of CMUT with nonaxisymmetric lateral venting channels by axisymmetric sealed cavity.

\section{ACKNOWLEDGMENT}

This work has been supported by the Labex ACTION project (contract "ANR-11-LABX-01-01").

\section{REFERENCES}

[1] B. T. Khuri-Yakub, and Ö. Oralkan, "Capacitive micromachined ultrasonic transducers for medical imaging and therapy," Journal of micromechanics and microengineering, 2011, vol. 21, no 5, p. 054004.

[2] N. Apte, K. K. Park, and B. T. Khuri-Yakub, "Finite element analysis of CMUTs with pressurized cavities," 2012 IEEE International Ultrasonics Symposium. IEEE, 2012. p. 979-982.

[3] K. K. Park, et al. "Optimum design of circular CMUT membranes for high quality factor in air," 2008 IEEE Ultrasonics Symposium. IEEE, 2008. p. 504-507.

[4] M. Bao, and H. Yang, "Squeeze film air damping in MEMS," Sensors and Actuators A: Physical, 2007, vol. 136, no 1, p. 3-27.

[5] A. Caronti, G. Caliano, A. Iula, and M. Pappalardo, "An accurate model for capacitive micromachined ultrasonic transducers," IEEE Transactions on Ultrasonics, Ferroelectrics, and Frequency Control, 2002, vol. 49, no 2, p. 159-168.

[6] R. B. Darling, C. Hivick, and J. Xu, "Compact analytical modeling of squeeze film damping with arbitrary venting conditions using a Green's function approach," Sensors and Actuators A: Physical, 1998, vol. 70, no 1, p. 32-41.

[7] M. Bellaredj, et al. "Anodic bonding using SOI wafer for fabrication of capacitive micromachined ultrasonic transducers," Journal of Micromechanics and Microengineering, 2014, vol. 24, no 2, p. 025009.

[8] A. H. Nayfeh, and M. I. Younis, "A new approach to the modeling and simulation of flexible microstructures under the effect of squeeze-film damping," Journal of Micromechanics and Microengineering, 2003, vol. 14 , no 2, p. 170.

[9] M. A. Bıçak, and M. D. Rao, "Analytical modeling of squeeze film damping for rectangular elastic plates using Green's functions," Journal of Sound and Vibration, 2010, vol. 329, no 22, p. 4617-4633.

[10] B. Ahmad, and R.Pratap, "Analytical evaluation of squeeze film forces in a CMUT with sealed air-filled cavity," IEEE Sensors Journal, 2011, vol. 11 , no 10 , p. $2426-2431$.

[11] G. G. Yaralioglu, et al. "Calculation and measurement of electromechanical coupling coefficient of capacitive micromachined ultrasonic transducers," IEEE transactions on ultrasonics, ferroelectrics, and frequency control, 2003, vol. 50, no 4, p. 449-456.

[12] J. J. Blech, "On isothermal squeeze films," Journal of lubrication technology, 1983, vol. 105, no 4, p. 615-620..

[13] T. Veijola, H. Kuisma, J. Lahdenperä, and T. Ryhänen, "Equivalentcircuit model of the squeezed gas film in a silicon accelerometer," Sensors and Actuators A: Physical, 1995, vol. 48, no 3, p. 239-248.

[14] www.comsol.com 